\title{
MONOSYNAPTIC INHIBITION OF PHRENIC MOTONEURONS: A LONG DESCENDING PROJECTION FROM BÖTZINGER NEURONS ${ }^{1}$
}

\author{
E. G. MERRILL ${ }^{2}$ AND L. FEDORKO ${ }^{3}$ \\ University Laboratory of Physiology, Parks Road, Oxford OX1 3PT, United Kingdom \\ Received November 29, 1983; Revised February 22, 1981; Accepted February 24, 1984
}

\begin{abstract}
Synaptic connections between medullary expiratory neurons and phrenic motoneurons were studied in anesthetized cats using spike-triggered averaging of synaptic noise in phrenic motoneurons. Monosynaptic inhibition of phrenic motoneurons originating from the rostral medullary expiratory neurons of the Botzinger complex was revealed. Neither mono- nor disynaptic inhibition from expiratory neurons of the nucleus retroambigualis was found. It was concluded that Bötzinger complex expiratory neurons make long descending inhibitory connections with phrenic motoneurons: the expiratory neurons of the nucleus retroambigualis do not appear to make synaptic connections with phrenic motoneurons.
\end{abstract}

Phrenic motoneurons (PMs) are the last neurons to integrate the respiratory output to the diaphragm. In the past it was believed that synaptic inhibition during the expiratory phase in these neurons was weak, if it existed at all (Gill and Kuno, 1963). Zielinski and Gebber (1975), however, concluded that there is substantial synaptic inhibitory input of supraspinal origin to PMs during expiration. Their experiments also showed that this inhibition increases toward the end of expiration. Berger (1979) reported that at least $30 \%$ of PMs are actively inhibited during expiration. One possibility arising from these observations is that medullary expiratory neurons, located in the caudal parts of the nucleus retroambigualis (NRA), may provide this inhibition. More than $90 \%$ of medullary expiratory neurons have long spinal axons (Merrill, 1970), and they have a firing pattern with a time course similar to that of PM inhibition. However, previous anatomical studies (Merrill, 1970), using the antidromic mapping technique (Merrill, 1971; Jankowska and Roberts, 1972a), failed to reveal any axonal collaterals of these neurons at the level of the phrenic motor nucleus. On the other hand, these studies do not exclude the possibility of polysynaptic inhibition, or the possibility that the NRA expiratory axons give very short collaterals not revealed with the antidromic mapping method.

Recently, a new group of medullary expiratory neurons, located in the vicinity of the retrofacial nucleus, was described (Kalia et al. 1979; Lipski and Merrill, 1980). These neurons, called the Bötzinger complex (BOT) (Kalia, 1981), have a firing pattern similar to that of the expiratory NRA neurons (i.e., with peak activities late in expiration). BOT expiratory neurons exert monosynaptic inhibition on the inspiratory neurons of

\footnotetext{
${ }^{1}$ This work was supported by the Medical Research Council, United Kingdom, the Foundation for Sudden Infant Death, and the European Science Foundation.

${ }^{2}$ To whom correspondence should be addressed.

${ }^{3}$ Present address: Medical Academy, Institute of Physiology, 00-927 Warsaw, Poland.
}

the contralateral nucleus tractus solitarius (NTS) (Merrill et al., 1983). Further studies reveal not only rich BOT projections to other medullary inspiratory nuclei (NRA and ipsilateral NTS) but also descending spinal projections (Bianchi and Barillot, 1982; Fedorko, 1982; Fedorko and Merrill, 1984). The presence of spinal projections from these neurons, with axonal arbors at the phrenic motor nucleus level (Fedorko and Merrill, 1984), suggests that they may exert synaptic actions on PMs.

The present experiments were performed to verify this hypothesis, as well as to investigate the possibility of the disynaptic inhibition of PMs by NRA expiratory neurons. Spiketriggered averages (STAs) (Jankowska and Roberts, 1972b), triggered by the spikes of NRA and BOT expiratory cells, were used to reveal unitary postsynaptic potentials (PSPs) buried in the synaptic noise of PMs. This method can demonstrate both monosynaptic and disynaptic connections (Watt et al., 1976).

\section{Materials and Methods}

Experiments were performed on 15 cats anesthetized with sodium pentobarbital (Sagatal, M\&B, $45 \mathrm{mg} / \mathrm{kg}$, i.p.), supplemented by intravenous administration $(3$ to $5 \mathrm{mg} / \mathrm{kg} / \mathrm{hr}$ ). The animals were tracheostomized, paralyzed (Flaxidil, $10 \mathrm{mg} / \mathrm{kg} / \mathrm{hr}$, i.v.), artificially ventilated, and subjected to bilateral pneumothorax.

On one side, the C5 phrenic root was dissected laterally in the neck, covered with warm paraffin oil, and used for monitoring respiratory activity as well as for stimulation of PM axons.

The medulla oblongata was exposed through an occipital craniotomy and the cervical spinal cord (C4-C5 segment) was exposed through laminectomy at $\mathrm{C} 3-\mathrm{C} 6$. Extracellular unit recordings were made from the expiratory region of the NRA and BOT (contralateral to the dissected phrenic nerve root). Platinum-plated, glass-coated tungsten microelectrodes (Digitimer) were used for these recordings. Placement of the recording electrodes within the BOT region has been described in detail in previous reports (Merrill et al. 1983; Fedorko and Merrill, 1984).

Intracellular recordings from $\mathrm{PMs}$ were made with glass micropipettes filled with $2 \mathrm{M}$ potassium acetate (during two experiments, $2 \mathrm{M}$ $\mathrm{KCl}$ filled electrodes were used in an attempt to reverse the recorded IPSPs by chloride ion injection); DC resistance was 10 to 15 megohms. 
During search penetrations of the $\mathrm{C} 5$ spinal segment the phrenic (C5) nerve root was stimulated continuously $(2 \mathrm{~Hz}, 0.1 \mathrm{msec}, 30 \mu \mathrm{A})$, so that motoneurons could be identified by their antidromically evoked action potentials. If a penetrated motoneuron displayed spontaneous activity, hyperpolarizing or depolarizing current ( 2 to $4 \mathrm{nA}$ ) was passed through the recording electrode to inactivate the cell before averaging its membrane potential. Subsequently, the membrane potential recording was filtered (bandpass $1 \mathrm{~Hz}$ or $10 \mathrm{~Hz}$ to $5 \mathrm{kHz}$ ) and averaged. Spikes of the NRA or BOT expiratory neurons were discriminated and used to trigger averages. Signals were displayed on a cathode ray tube and photographed with a Polaroid camera.

\section{Results}

Forty-nine PMs were impaled during these experiments with stable membrane potentials above $45 \mathrm{mV}$ (range, 45 to $70 \mathrm{mV}$; mean, $58.2 \mathrm{mV}$ ); all showed a depolarizing shift of the membrane potential during inspiration, associated with increase of the synaptic noise visible on high gain $\mathrm{AC}$ traces. These inspiratory depolarizations had patterns similar to those of two of the categories previously described by Berger (1979). The first group has a slowly rising depolarization during inspiration (Berger's type "A") with a rapid depolarization at the end of inspiration (Fig. 1, top); the second group has a more rapid depolarization, with a visible membrane potential plateau during inspiration (Berger's type "B") and slower hyperpolarization at the end of inspiration (Fig. 1, middle). However, it has to be stressed that many motoneurons displayed intermediate membrane potential patterns. Only seven motoneurons had obvious inhibitory synaptic potentials (i.e., easily visible on high gain AC traces). 'I'wo of three PMs recorded with KCI recording electrodes (10 megohms) had prominent depolarizing membrane potential waves during expiration, after about $5 \mathrm{~min}$ of $\mathrm{Cl}^{-}$infusion. These waves were similar to those caused by IPSP reversal resulting from chloride ion leakage into the cells (Fig. 2, bottom) (Berger, 1979).

Synaptic action of BOT expiratory neurons on PMs. The activities of $16 \mathrm{BOT}$ expiratory neurons were recorded; all of them displayed fairly high firing rates during expiration (up to $127 \mathrm{~Hz}$ ) (Fig. 3A). These BOT neurons were used to trigger averages on membrane synaptic noise in 32 simultaneously recorded PMs.

In all, 37 BOT-PM pairs were studied. Twelve of the 37 averages showed hyperpolarizing shifts correlated with the action potentials of the triggering BOT neuron. These hyperpolarizations had shapes (Fig. $4 A$ ) resembling the unitary
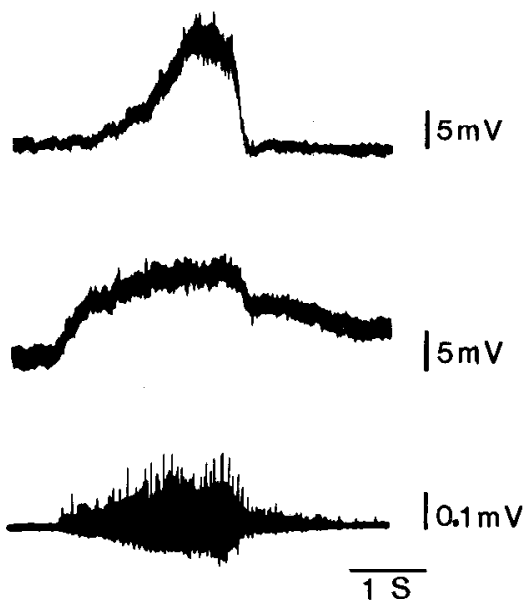

Figure 1. Membrane potential DC shifts in PMs. The top trace shows an example of a type A shift (Berger, 1979). The middle trace shows a type $\mathrm{B}$ shift in another motoneuron. The bottom trace is the phrenic neurogram. The two DC shifts were recorded a few minutes apart in the same animal; identical respiratory cycles were selected from film strips and aligned for the figure.
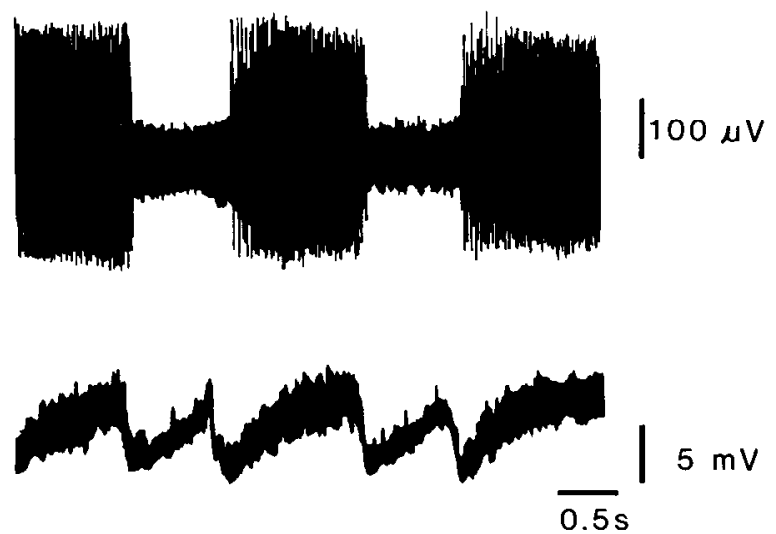

Figure 2. Chloride reversal of the expiratory inhibitory shift in a PM. The top trace shows a multiunit recording from expiratory neurons of BOT; the bottom trace shows the slow membrane potential shifts in a PM approximately 5 min after impalement with a KCl-filled microelectrode. Note the prominent depolarization and large amplitude synaptic noise during expiration.

A

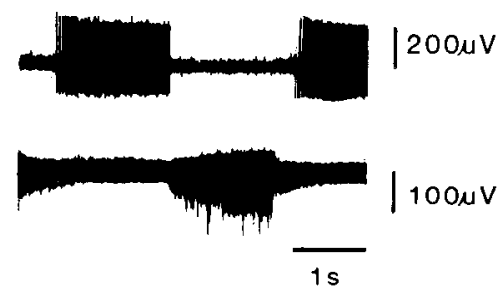

B
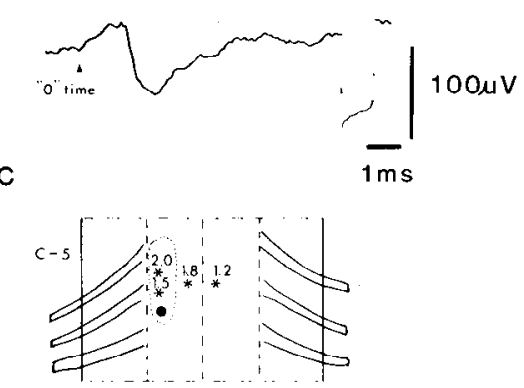

$$
\overline{1 \mathrm{~mm}}
$$

Figure 3. BOT spike train, unitary IPSP, and antidromic latency map. $A$ shows (top) the spike train from a BOT expiratory neuron and (bottom) the associated phrenic neurogram. $B$ shows the unitary IPSP revealed by STA in a PM (1024 sweeps, sweep delayed 1 msec with a delay line). $C$ shows penetration sites of a monopolar stimulating electrode, subsequent to obtaining the IPSP in $B$. The recording site was at the solid circle. The antidromic latencies obtained in the BOT expiratory neuron are shown above the stimulus penetrations (asterisks).

IPSPs recorded by others in various CNS neurons (Jankowska and Roberts, 1972b; Rapoport et al., 1977; Hikosaka et al., 1978). IPSP amplitudes ranged from 20 to $100 \mu \mathrm{V}$ (mean, 47.7 $\mu \mathrm{V}$ ). Time to peak (measured from $10 \%$ to $90 \%$ of amplitude) varied from 0.54 to $1.4 \mathrm{msec}$ (mean, $0.71 \mathrm{msec}$ ). In IPSPs with a smooth decay phase $(N=6)$, the half-widths (Jack et al., 1971 ) varied from 1.6 to $3.2 \mathrm{msec}$ (mean, $2.2 \mathrm{msec}$ ). IPSPs had latencies from 1.3 to $3.0 \mathrm{msec}$ (mean, $1.9 \mathrm{msec}$ ). In one experiment an attempt was made to average synaptic potentials using $\mathrm{KCl}$ electrodes. We were not fortunate enough to record the same PSP both before and after $\mathrm{Cl}^{-}$reversal, but progressive 

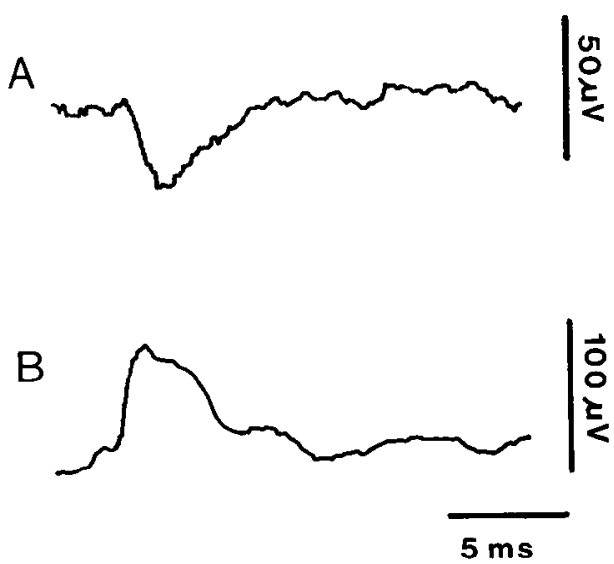

Figure 4. STAs of unitary PSPs. A, A unitary IPSP (512 sweeps). B, A reversed IPSP from the motoneuron shown in Figure 2, after chloride IPSP reversal (1024 sweeps). $A$ and $B$ were recorded in different animals.

reversals of the expiratory waves such as that shown in Figure 2 were continuously monitored. Following reversal, the PSPs (seen in single oscilloscope sweeps) making up the synaptic wave had clearly changed from hyperpolarizing potentials to depolarizing potentials. The PSP shown in Figure $4 B$ was recorded following the reversal of the expiratory synaptic wave; we believe it to be a reversed IPSP. No other depolarizing PSPs were ever seen synchronized with BOT action potentials.

Several BOT neurons were held long enough to be used as triggers for ST $\Lambda$ s in more than one PM. In these multiple STA series, divergence seemed to differ markedly between individual BOTs. Thus, for example, three series showed $0 \%$ (0 IPSPs in 6 PMs tested), $20 \%$ ( 1 in 5) and 100\% (3 in 3) projections. Since we did not check each BOT for a spinal axon, it is not clear how to assess the apparent absence of inputs from some BOTs to PMs, or, further, whether the wide divergence seen for others is merely a consequence of irregular sampling.

All averages were made using randomly chosen neuronal pairs. Axonal projections for the BOT neurons used for STA triggering were not determined prior to averaging to avoid damage to the spinal cord; both the edema and lesions caused by multiple penetrations of even very fine tungsten electrodes might have reduced the likelihood of observing PSPs. In one case, however, after the inhibitory synaptic potential was seen in the averaged membrane potential of a PM (Fig. $3 B$ ), an attempt to locate the descending axon of the triggering BOT neuron (Fig. $3 A$ ) was made. Surprisingly the expiratory neurone could not be activated from the region of the intracellular penetration. Successful antidromic activation was obtained from more rostral locations (but with longer latencies than the IPSP itself) and at short latencies from the spinal cord contralateral to the intracellular penetration iste (Fig. $3 \mathrm{C}$ ). This finding indicates either that the arbors of the descending BOT axons crossed the midline (Fedorko and Merrill, 1984) at the level of the phrenic motor nucleus or that the dendritic tree of the PM extended across the midline (Cameron et al., 1983), possibly receiving bilateral synaptic inputs.

Synaplic action of the NRA expiratory neurons on phrenic motoneurons. Seventeen NRA expiratory neurons were recorded and used to trigger membrane potential averages in 17 PMs. In all, 19 NRA-PM pairs were studied for the presence of synaptic connections. No synaptic potentials were revealed (the resolution was better than $5 \mu \mathrm{V}, 1024$ to 2048 averaged sweeps). The size of the sample, although smaller, is comparable with the assessment described above for BOT-PM connections. We conclude that there are no strong mono- or disynaptic connections between the expiratory neurons of the NRA and PMs.

\section{Discussion}

Present experiments have shown that the synaptic inhibition of PMs originates, at least in part, from the medullary expira tory neurons of the BOT complex. The recorded unitary IPSPs had short rise times and amplitudes which are consistent with them being monosynaptic. From earlier studies (Berger, 1979), as well as from the present experiments, it is apparent that strong expiratory inhibition is not present in all PMs. Indeed, in some neurons it was impossible to discover any synaptic noise during expiration; even long injections of chloride in one PM failed to produce obvious changes in the membrane potential pattern during expiration. On the other hand, in the neurons which displayed some synaptic activity in expiration, even a relatively small chloride efflux from the recording electrodes (without passing an ejecting current) caused an obvious reversal of the normally occurring expiratory hyperpolarization. These findings, and the presence of synaptic inhibition in 12 of 37 $(32 \%)$ of randomly chosen neuronal BOT-PM pairs, indicate that much of the expiratory inhibition probably originates from BOT expiratory neurons.

As was described in previous studies (Merrill et al., 1983), BOT expiratory neurons inhibit the medullary inspiratory neurons of the NTS. These NTS neurons are last-order interneurons for PMs (Cohen et al., 1974; Hilaire and Monteau, 1976; Fedorko et al., 1983). The presence of rich, bilateral spinal projections from BOT neurons (Fedorko and Merrill, 1984) must enhance their effectiveness as a common source of inhibition of the inspiratory motor output to the diaphragm during expiration, on both the medullary and spinal levels.

The absence in our sample of any synaptic potentials in PMs correlated with the activity of the expiratory neurons of the NRA suggests a lack of synaptic connections between these two neuronal populations. This observation is consistent with the apparent absence of terminal arborizations from NRA expiratory neurons in lower cervical cord (Merrill, 1970). It is possible that NRA expiratory neurons contribute to the synaptic inhibition of PMs via polysynaptic pathways. However, disynaptic NRA influences perhaps would be detected (if they exist) by STA methods (Watt et al., 1976). A polysynaptic inhibitory pathway, on the other hand, would require more than one additional expiratory population of expiratory interneurons having similar patterns of activity and projecting to or located in the spinal cord. At present, no such neuronal populations have been described. The most likely explanation, therefore, is that NRA expiratory neurons do not contribute to the expiratory inhibition of PMs in the cat, and that BOT ncurons are the main source of this inhibition.

\section{References}

Berger, A. J. (1979) Phrenic motoneurons in the cat: Subpopulations and nature of respiratory drive potentials. J. Neurophysiol. 42: 7690.

Bianchi, A. L., and J. C. Barillot (1982) Respiratory neurons in the region of the Retrofacial Nucleus: Pontile, medullary, spinal and vagal projections. Neurosci. Lett. 31: 277-282.

Cameron, W. E., D. B. Averill, and A. J. Berger (1983) Morphology of cat phrenic motoneurons as revealed by intracellular injection of horseradish peroxidase. J. Comp. Neurol. 219: 70-80.

Cohen, M. I., M. F. Piercey, P. M. Gootman, and P. Wolotsky (1974) Synaptic connections between medullary inspiratory neurons and phrenic motoneurons as revealed by crosscorrelation. Brain Res. 81: 319-324.

Fedorko, L. (1982) Axonal projections from Bötzinger expiratory neurones to other medullary nuclei and spinal cord in the cat. J. Physiol. (Lond.) 332: 80P.

Fedorko, L., and E. G. Merrill (1984) Axonal projections from rostral expiratory neurones of the Bötzinger complex to medulla and spinal cord in the cat. J. Physiol. (Lond.) 350: 487-496.

Fedorko, L., E. G. Merrill, and J. Lipski (1983) Two descending medullary inspiratory pathways to phrenic motoneurones. Neurosci. Lett. 43: 285-291. 
Gill, P. K., and M. Kuno (1963) Excitatory and inhibitory actions on phrenic motoneurones. J. Physiol. (Lond.) 168: 274-289.

Hikosaka, O., Y. Igusa, S. Nakao, and H. Shimazu (1978) Direct inhibitory synaptic linkage of pontomedullary reticular burst neurons with abducens motoneurons in the cat. Exp. Brain Res. 33: 337.

Hilaire, G., and R. Monteau (1976) Connexions entre les neurones inspiratoires bulbaires et les motoneurones phréniques et intercostaus. J. Physiol. (Paris) 72: 987-1000.

Jack, J. J. B., S. Miller, R. Porter, and S. J. Redman (1971) The time course of minimal excitatory post-synaptic potentials evoked in spinal motoneurones by group Ia afferent fibers. J. Physiol. (Lond.) 215: $353-380$.

Jankowska, E., and W. J. Roberts (1972a) An electrophysiological demonstration of the axonal projections of single spinal interneurones in the cat. J. Physiol. (Lond.) 222: 597-622.

Jankowska, E., and W. J. Roberts (1972b) Synaptic actions of interneurones mediating reciprocal Ia inhibition of motoneurones. $J$. Physiol. (Lond.) 222: 623-642.

Kalia, M. (1981) Anatomical organization of central respiratory neurons. Annu. Rev. Physiol. 43: 105-120.

Kalia, M., J. L. Feldman, and M. I. Cohen (1979) Afferent projection to the inspiratory neuronal region of the ventrolateral nucleus of the tractus solitarius in the cat. Brain Res. 171: 135-141.
Lipski, J., and E. G. Merrill (1980) Electrophysiological demonstration of the projection from expiratory neurones in rostral medulla to contralateral dorsal respiratory group. Brain Res. 197: 521-524.

Merrill, E. G. (1970) The lateral respiratory neurones of the medulla: Their association with nucleus ambiguus, nucleus retroambigualis, the spinal accessory nucleus and spinal cord. Brain Res. 24: 11-28.

Merrill, E. G. (1971) The descending pathway from the lateral respiratory neurones in cats. J. Physiol. (Lond.) 218: 82-83P.

Merrill, E. G., J. Lipski, L. Kubin, and L. Fedorko (1983) Origin of expiratory inhibition of nucleus tractus solitarius inspiratory neurones. Brain Res. 263: 43-50.

Rapoport, S., A. Susswein, Y. Uchino, and V. J. Wilson (1977) Synaptic actions of individual vestibular neurones on cat neck motoneurones. J. Physiol. (Lond.) 272: 367-382.

Watt, D. I. D., E. K. Stauffer, A. Taylor, R. M. Reinking, and D. G. Stuart (1976) Analysis of muscle receptor connections by spiketriggered averaging. I. Spindle primary and tendon organ afferents. J. Neurophysiol. 39: 1375-1392.

Zielinski, A. T., and G. L. Gebber (1975) Basis for late expiratory spinal inhibition of phrenic discharge. Am. J. Physiol. 228: 16901694 